\title{
On backstepping boundary control for a class of linear port-Hamiltonian systems
}

\author{
Hector Ramirez, Hans Zwart, Yann Le Gorrec and Alessandro Macchelli
}

\begin{abstract}
Backstepping boundary control is investigated for a class of linear port-Hamiltonian systems. It is shown that by considering as target system an exponentially stable dissipative PHS, i.e., a PHS with a linear dissipation term and homogeneous boundary conditions, a coordinate transformation based on a multiplicative operator suffices to map the open-loop system into the target system. The condition for the existence of the transformation is algebraic. Hence, the backstepping transformation and the associated matching condition are simpler than the conventional ones that considers Volterra integral terms and kernel conditions in the form of partial differential equations. Since the controller has been developed for a general class of linear PHS it is applicable to a large class of physical systems, as for instance transport, beam and wave equations. The result is illustrated on the examples of a transport equation and a vibrating string on a 1D spatial domain.
\end{abstract}

\section{INTRODUCTION}

Many physical systems described by partial differential equations (PDEs) are controlled through their boundaries. This is for instance the case for transmission lines, flexible beams and plates, tubular and nuclear fusion reactors and so on. This class of systems is called Boundary Controlled Systems (BCS) [1].

Backstepping boundary control of PDEs [2], [3], [4] has proven to be a powerful design method for the boundary feedback stabilization of infinite dimensional systems. It has been succuesfully applied to reaction, convection, diffusion, transport and wave equations [5], [6], [7], [8], [9]. The synthesis of a backstepping controller consists in mapping the controlled system into an exponentially stable target system, i.e., mapping the PDE into a target PDE which is known to be exponential stable. The key step in the design procedure is to find an invertible Volterra integral coordinate transformation. To this end the kernel functions of the transformation should satisfy a matching equation, in the form of a PDE, whose solution is then used to compute the controller. Even though the backsteeping approach is a

H. Ramirez and Y. Le Gorrec are with the Université Bourgogne FrancheComté (UBFC) and FEMTO-ST institute, AS2M department, 24 rue Alain Savary, 25000 Besançon, France (e-mails: hector.ramirez@femto-st.fr and yann.le.gorrec@ens2m.fr)

H. Zwart is with the University of Twente, Faculty of Electrical Engineering, Mathematics and Computer Science, Department of Applied Mathematics, P.O. Box 2177500 AE Enschede, The Netherlands. (email: h.j.zwart@utwente.nl), and the Technische Universiteit Eindhoven, Department of Mechanical Engineering, P.O. Box 513, 5600 MB Eindhoven, The Netherlands. (e-mail: h.j.zwart@tue.nl)

A. Macchelli is with the Department of Electrical, Electronic and In formation Engineering Guglielmo Marconi (DEI), University of Bologna, viale del Risorgimento 2, 40136 Bologna, Italy. (e-mail: alessandro.macchelli@unibo.it) practical tool for boundary control design its difficulty lays in finding a solution for the kernel matching equations. Usually this is done in a case by case basis for different classes of PDEs and since the coordinate transformation is of integral nature it is not a trivial task [4].

In the last decade, an alternative approach has been developed in order to deal with a large class of physical systems. This approach is based on the extension of the Hamiltonian formulation to open infinite dimensional systems [10]. In the $1 \mathrm{D}$ linear case it gave rise to the definition of boundary controlled port-Hamiltonian systems [11] and allowed to parametrize all the possible boundary conditions that define a boundary control system by using simple matrix conditions [12]. Many variations around these primary works can be found in [13] and in [14]. Well possessedness and stability have been investigated in open-loop and in the case of static boundary feedback control in [15] and [16], [17], respectively, and in the case of dynamic linear control in [18], [19]. More recently there have been efforts in developing systematic control design approaches, as for instance the ones based on Casimir functions [20].

In this paper a backstepping approach is used to develop a boundary controller for linear PHS with constant parameters. The target system is a dissipative PHS, i.e., a PHS with an in-domain dissipation term and homogenoeus boundary conditions. A general class of coordinate transformation is then considered and by diagonalizing the open-loop and the target systems it is deduced that a linear multiplicative coordinate transformation suffices to achieve an invertible mapping between the open-loop and the target systems. The condition for the existence of the transformation is an algebraic equation on the boundaries. Hence the transformation and the conditions for its existence are simpler than in standard backstepping. The coordinate transformation is then used to compute the boundary controller. Since the controller has been developed for a general linear PHS it is applicable to a large class of physical systems, as for instance transport, beam and wave equations.

The paper is organized as follows. In Section II we give the preliminaries on the considered class of PHS. Section III presents the main result of the paper, namely the backstepping boundary controller. In Section IV we present two examples, a transport equation and a wave equation. Finally in Section V we give some final comments and discussions on future work. 


\section{A CLASS OF PORT-HAMILTONIAN SYSTEMS}

The systems under study are described by the following 1-D partial differential equation (PDE):

$$
\frac{\partial \tilde{x}}{\partial t}(t, \zeta)=P_{1} \frac{\partial}{\partial \zeta}(\tilde{x}(t, \zeta))
$$

$\zeta \in(0,1)$, where $P_{1} \in M_{n}(\mathbb{R})^{1}$ is a non-singular symmetric matrix, and $\tilde{x}$ takes values in $\mathbb{R}^{n}$.

For the above PDE we assume that some boundary conditions are homogeneous, whereas others are controlled. Thus there are matrices of appropriate sizes such that

$$
u(t)=W_{B, 1}\left[\begin{array}{l}
\tilde{x}(t, 1) \\
\tilde{x}(t, 0)
\end{array}\right]
$$

and

$$
0=W_{B, 2}\left[\begin{array}{l}
\tilde{x}(t, 1) \\
\tilde{x}(t, 0)
\end{array}\right]
$$

Furthermore, there is a boundary output given by

$$
y(t)=W_{C}\left[\begin{array}{l}
\tilde{x}(t, 1) \\
\tilde{x}(t, 0)
\end{array}\right] .
$$

To study the existence and uniqueness of solution to the above controlled PDE, we follow the semigroup theory, see also [12], [14]. Therefore we define the state space $X$ as $X=L_{2}\left((0,1) ; \mathbb{R}^{n}\right)$ with the standard inner product $\left\langle\tilde{x}_{1}, \tilde{x}_{2}\right\rangle$ and norm $\|\tilde{x}\|^{2}=\langle\tilde{x}, \tilde{x}\rangle$. Note that the norm on $X$ and the $L_{2}$ norm are equivalent. Hence $X$ is a Hilbert space. The reason for selecting this space is that $\|\cdot\|^{2}$ is related to the energy function of the system, i.e., the total energy of the system equals $E(t)=\frac{1}{2}\|\tilde{x}\|^{2}$. The Sobolev space of order $k$ is denoted by $H^{k}\left((0,1), \mathbb{R}^{n}\right)$.

Associated to the (homogeneous) pde we define the operator $A \tilde{x}=P_{1}(\partial / \partial \zeta)(\tilde{x})$ with domain

$$
D(A)=\left\{\tilde{x} \in H^{1}\left((0,1) ; \mathbb{R}^{n}\right) \mid\left[\begin{array}{c}
\tilde{x}(1) \\
\tilde{x}(0)
\end{array}\right] \in \operatorname{ker} W_{B}\right\}
$$

where $W_{B}=\left[\begin{array}{l}W_{B, 1} \\ W_{B, 2}\end{array}\right]$. For the rest of the paper we make the following hypothesis

Assumption 2.1: For the operator $A$ and the pde (1)-(4) the following hold:

1) The matrix $W_{B}$ is an $n \times 2 n$ matrix of full rank;

2) For $x_{0} \in D(A)$ we have $\left\langle A \tilde{x}_{0}, \tilde{x}_{0}\right\rangle \leq 0$.

3) The number of inputs and outputs are the same, $k$, and for classical solutions of (1)-(4) there holds $\dot{E}(t)=$ $u(t)^{\top} y(t)$.

We remark that from hypothesis (1) and (2) it follows that the system (1)-(4) is a boundary control system (see [12], [14], [21]), and so for $u \in C^{2}\left([0, \infty) ; \mathbb{R}^{k}\right), \tilde{x}(0) \in$ $H^{1}\left((0,1) ; \mathbb{R}^{n}\right)$, satisfying (2) and (3) (for $\left.t=0\right)$, there exists a unique classical solution to (1)-(4). Thus for these dense sets of initial conditions and inputs hypothesis (3) makes sense.

\footnotetext{
${ }^{1} M_{n}(\mathbb{R})$ denote the space of real $n \times n$ matrices
}

\section{THE BACKSTEPPING CONTROLLER}

Now we shall construct a stabilizing controller for the port-Hamiltonian system introduced in the previous section. The main idea is to map the PHS (1) into an exponentially stable target system given by a dissipative PHS. A coordinate transformation is then considered, which is more general than the Volterra integral transformation usually used in the backstepping design procedure. This permits to systematically derive a transformation which turns out to be based on a multiplicative operator. An algebraic matching condition has to be satisfied to guarantee the invertibility of the transformation. This is a simpler condition than the usual kernel conditions in standard backstepping.

Before presenting the main result of the paper we introduce some notation. Since $P_{1}$ is symmetric, there exists a matrix $T \in M_{n}(\mathbb{R})$ such that $T^{-1} P_{1} T$ is diagonal. We denote this diagonal matrix by $\Lambda$, i.e.,

$$
\Lambda=T^{-1} P_{1} T \text {. }
$$

Since $P_{1}$ is invertible, $\Lambda$ cannot have a zero element on the diagonal. The columns of $T$ consists of the eigenvectors of $P_{1}$ and the eigenvalues of $P_{1}$ form the diagonal of $\Lambda$, i.e., $\Lambda=\operatorname{diag}\left(\lambda_{i}\right)$.

The main result of this section is the following proposition.

Proposition 3.1: Beside the notation as introduced above, we define for $c>0$

$$
\mathcal{M}(\zeta)=\left[\begin{array}{ccc}
e^{\frac{c}{\lambda_{1}} \zeta} & \cdots & 0 \\
\vdots & \ddots & \vdots \\
0 & \cdots & e^{\frac{c}{\lambda_{n}} \zeta}
\end{array}\right]
$$

If there exists an invertible, diagonal matrix $\mathcal{A}$ such that for all $p, q \in \mathbb{R}^{n}$ satisfying

$$
\left[\begin{array}{l}
p \\
q
\end{array}\right] \in \operatorname{ker}\left(W_{B, 2}\right)
$$

we have that

$$
\left[\begin{array}{c}
T \mathcal{A M}(1) T^{-1} p \\
T \mathcal{A} T^{-1} q
\end{array}\right] \in \operatorname{ker}\left(\left[\begin{array}{l}
W_{B, 1} \\
W_{B, 2}
\end{array}\right]\right),
$$

then the boundary controller

$$
u(t)=-W_{B, 1}\left[\begin{array}{c}
T(\mathcal{A} M(1)-I) T^{-1} \tilde{x}(t, 1) \\
T(\mathcal{A}-I) T^{-1} \tilde{x}(t, 0)
\end{array}\right] .
$$

exponentially stabilizes the PHS (1). Furthermore, there exists a constant $m_{0}$ such that solutions of the closed loop system satisfy

$$
\|\tilde{x}(t)\| \leq m_{0} e^{-c t}\|\tilde{x}(0)\| .
$$

Proof: Defining $x=T^{-1} \tilde{x}$ and using (5) we can rewrite (1) as

$$
\frac{\partial x}{\partial t}=\Lambda \frac{\partial}{\partial \zeta}(x(t, \zeta))
$$

The boundary conditions are formulated in terms of $x$ as follows

$$
u(t)=\tilde{W}_{B, 1}\left[\begin{array}{l}
x(t, 1) \\
x(t, 0)
\end{array}\right]
$$


and

$$
0=\tilde{W}_{B, 2}\left[\begin{array}{l}
x(t, 1) \\
x(t, 0)
\end{array}\right] .
$$

where $\tilde{W}_{B, 1}=W_{B, 1}\left[\begin{array}{cc}T & 0 \\ 0 & T\end{array}\right]$ and $\tilde{W}_{B, 2}=W_{B, 2}\left[\begin{array}{cc}T & 0 \\ 0 & T\end{array}\right]$.

Choose as target system

$$
\frac{\partial \tilde{z}}{\partial t}=P_{1} \frac{\partial \tilde{z}}{\partial \zeta}-c I \tilde{z}
$$

where $c \in \mathbb{R}, c>0$, with homogeneous boundary conditions

$$
0=\left[\begin{array}{l}
W_{B, 1} \\
W_{B, 2}
\end{array}\right]\left[\begin{array}{l}
\tilde{z}(t, 1) \\
\tilde{z}(t, 0)
\end{array}\right]
$$

Using the first two items in Assumption 2.1 it is straightforward to show that this target system is exponentially stable as soon as $c>0$. Furthermore, for smooth initial conditions

$$
\frac{d}{d t}\|z(t)\|^{2} \leq-2 c\|z(t)\|^{2}
$$

Thus for all solutions we have

$$
\|z(t)\| \leq e^{-c t}\|z(0)\| .
$$

Using (5) the target system can equivalently be formulated as the diagonal target system

$$
\frac{\partial z}{\partial t}=\Lambda \frac{\partial z}{\partial \zeta}-c I z
$$

with homogeneous boundary conditions

$$
0=\left[\begin{array}{l}
\tilde{W}_{B, 1} \\
\tilde{W}_{B, 2}
\end{array}\right]\left[\begin{array}{l}
z(t, 1) \\
z(t, 0)
\end{array}\right]
$$

We shall now prove that (7) defines a mapping of the diagonal representation (8)-(10) into (14)-(15).

Consider the coordinate transformation

$$
z(t, \zeta)=x(t, \zeta)+Q(x(t, \zeta))
$$

where $Q$ is a bounded linear mapping from $L^{2}\left((0,1) ; \mathbb{R}^{n}\right)$ to $L^{2}\left((0,1) ; \mathbb{R}^{n}\right)$.

On the one hand, by taking the partial derivative with respect to time one obtains

$$
\frac{\partial z}{\partial t}=\frac{\partial x}{\partial t}+Q\left(\frac{\partial x}{\partial t}\right)=\Lambda \frac{\partial x}{\partial \zeta}+Q\left(\Lambda \frac{\partial x}{\partial \zeta}\right)
$$

where we have used that $Q$ does not depend on $t$ and that it is linear. On the other hand by combining (14) and (16)

$$
\frac{\partial z}{\partial t}=\Lambda \frac{\partial z}{\partial \zeta}-c z=\Lambda\left[\frac{\partial x}{\partial \zeta}+\frac{\partial Q(x)}{\partial \zeta}\right]-c x-c Q(x),
$$

which implies that the following matching equation should be satisfied

$$
Q\left(\Lambda \frac{\partial x}{\partial \zeta}\right)=\Lambda \frac{\partial Q(x)}{\partial \zeta}-c x-c Q(x)
$$

In order to solve the above equation, we first try to solve it for special $x$. We choose these spacial $x$ of the form $x(\zeta)=$ $e^{s \zeta} v$, with $s \in \mathbb{C}, v \in \mathbb{C}^{n}$. Replacing this solution in (17) and using the linearity of $Q$ we obtain

$$
s Q\left(\Lambda e^{s \zeta} v\right)=\Lambda\left(\frac{\partial Q\left(e^{s \zeta} v\right)}{\partial \zeta}\right)-c e^{s \zeta} v-c Q\left(e^{s \zeta} v\right)
$$

Considering the particular solution given by $v_{1}=$ $\left[\begin{array}{llll}1 & 0 & \ldots & 0\end{array}\right]^{\top},(18)$ becomes

$$
s \lambda_{1} Q\left(e^{s \zeta} v_{1}\right)=\Lambda\left(\frac{\partial Q\left(e^{s \zeta} v_{1}\right)}{\partial \zeta}\right)-c e^{s \zeta} v_{1}-c Q\left(e^{s \zeta} v_{1}\right)
$$

To compute $Q\left(e^{s \zeta} v_{1}\right)$ we write

$$
Q\left(e^{s \zeta} v_{1}\right)=\left[\begin{array}{c}
q_{s, 1}(\zeta) \\
p_{s, 1}(\zeta) \\
\vdots \\
p_{s, n-1}(\zeta)
\end{array}\right]
$$

Using this notation the first line of (19) becomes

$$
s \lambda_{1} q_{s, 1}(\zeta)=\lambda_{1} \frac{\partial q_{s, 1}}{\partial \zeta}(\zeta)-c e^{s \zeta}-c q_{s, 1}(\zeta)
$$

and by rearranging

$$
\frac{\partial q_{s, 1}}{\partial \zeta}(\zeta)=q_{s, 1}(\zeta)\left(s+\frac{c}{\lambda_{1}}\right)+\frac{c}{\lambda_{1}} e^{s \zeta},
$$

which implies that

$$
q_{s, 1}(\zeta)=\alpha_{s, 1} e^{\left(s+\frac{c}{\lambda_{1}}\right) \zeta}-e^{s \zeta}
$$

for some constant $\alpha_{s, 1} \in \mathbb{C}$. The second line of (19) reads

$$
s \lambda_{1} p_{s, 1}(\zeta)=\lambda_{2} \frac{\partial p_{s, 1}}{\partial \zeta}(\zeta)-c p_{s, 1}(\zeta)
$$

which admits as solution $p_{s, 1}=0$. The following lines of (19) admit the same solution, hence we find that

$$
Q\left(e^{s \zeta} v_{1}\right)=\left[\begin{array}{c}
q_{s, 1}(\zeta) \\
0 \\
\vdots \\
0
\end{array}\right]
$$

We see that we can argue similarly for the other basis vectors of $\mathbb{R}^{n}$. Thus using the linearity of $Q$ once more, we obtain

$$
Q\left(e^{s \zeta} v\right)=\left[\begin{array}{cccc}
q_{s, 1}(\zeta) & 0 & \cdots & 0 \\
0 & q_{s, 2}(\zeta) & \cdots & 0 \\
\vdots & \ddots & \ddots & \vdots \\
0 & \cdots & 0 & q_{s, n}(\zeta)
\end{array}\right] v
$$

with $q_{s, i}(\zeta)=\alpha_{s, i} e^{\left(s+\frac{c}{\lambda_{i}}\right) \zeta}-e^{s \zeta}$, with $i=1, \ldots, n$. Imposing $\alpha_{s, i} \equiv \alpha_{i} \in \mathbb{R}$ the following factorization follows

$$
Q\left(e^{s \zeta} v\right)=\left[\begin{array}{ccc}
\alpha_{1} e^{\frac{c}{\lambda_{1}} \zeta}-1 & \cdots & 0 \\
\vdots & \ddots & \vdots \\
0 & \cdots & \alpha_{n} e^{\frac{c}{\lambda_{1}} \zeta}-1
\end{array}\right] e^{s \zeta} v
$$

So for $x(\zeta)=e^{s \zeta} v$ we see that

$$
(Q(x))(\zeta)=\operatorname{diag}\left(\alpha_{i}\left(e^{\frac{c}{\lambda_{i}} \zeta}-1\right) x(\zeta) .\right.
$$

Since $Q$ is linear and the subspace spanned by exponential functions lie dense in $L^{2}\left((0,1) ; \mathbb{R}^{n}\right)$, we find that any $Q$ which transforms (8) into (11) must be a multiplicative operator. Thus the transformation (16) becomes

$$
z(t, \zeta)=M(\zeta) x(t, \zeta)
$$


with

$$
M(\zeta)=\left[\begin{array}{ccc}
\alpha_{1} e^{\frac{c}{\lambda_{1}} \zeta} & \cdots & 0 \\
\vdots & \ddots & \vdots \\
0 & \cdots & \alpha_{n} e^{\frac{c}{\lambda_{1}} \zeta}
\end{array}\right]
$$

It remains to show that (16) is invertible, and that boundary conditions can be satisfied. For this we write $M$ as

$$
M(\zeta)=\mathcal{A M}(\zeta)
$$

with $\mathcal{A}=\operatorname{diag}\left(\alpha_{i}\right)$. It is straightforward to see that $M$ is invertible if and only if all alpha's are non-zero or equivalently $\mathcal{A}$ is invertible.

The boundary conditions of the target system are given by

$$
0=\left[\begin{array}{l}
W_{B, 1} \\
W_{B, 2}
\end{array}\right]\left[\begin{array}{l}
\tilde{z}(1) \\
\tilde{z}(0)
\end{array}\right]
$$

Recalling that $\tilde{z}=T z, z=\mathcal{A M} x$, and $x=T^{-1} \tilde{x}$ we can rewrite the above as

$$
0=\left[\begin{array}{l}
W_{B, 1} \\
W_{B, 2}
\end{array}\right]\left[\begin{array}{c}
T \mathcal{A M}(1) T^{-1} \tilde{x}(1) \\
T \mathcal{A} T^{-1} \tilde{x}(0)
\end{array}\right] .
$$

By the assumptions in the proposition, there exists an $\mathcal{A}$ such that the above holds for all states $\tilde{x}$ satisfying the boundary condition (3). So we can transform (boundedly and invertible) our system into the target system. From the first line of (24) we have that

$$
\begin{aligned}
0 & =W_{B, 1}\left[\begin{array}{c}
T \mathcal{A M}(1) T^{-1} \tilde{x}(1) \\
T \mathcal{A} T^{-1} \tilde{x}(0)
\end{array}\right] \\
& =W_{B, 1}\left[\begin{array}{l}
\tilde{x}(1) \\
\tilde{x}(0)
\end{array}\right]+W_{B, 1}\left[\begin{array}{c}
T(\mathcal{A M}(1)-I) T^{-1} \tilde{x}(1) \\
T(\mathcal{A}-I) T^{-1} \tilde{x}(0)
\end{array}\right] \\
& =u(t)+W_{B, 1}\left[\begin{array}{c}
T(\mathcal{A} \mathcal{M}(1)-I) T^{-1} \tilde{x}(1) \\
T(\mathcal{A}-I) T^{-1} \tilde{x}(0)
\end{array}\right] .
\end{aligned}
$$

From this the (stabilizing) input follows.

Since there are only (invertible) state transformation used between the port-Hamiltonian systems (1) and the target system the closed loop has the same decay rate as the target systems. This concludes the proof.

Remark 3.2: If (3) is absent, thus we have $n$ controls, then the conditions in the proposition are automatically satisfied, and so this system is exponentially stabilizable.

Remark 3.3: Notice that the target system (11) is a PHS with dissipation [14], with dissipation matrix $G_{0}=c I>0$.

\section{EXAMPLES}

In this section we shall illustrate the proposed controller on two classical examples, the transport equation and the clamped vibrating string (wave equation). Even though booth equations are of different nature, using the PHS formalism they belong to the same class of system and can hence be controlled in the same way. This is one of the main advantages of PHS based control design, booth in finite dimension and infinite dimensions [22], [14].

\section{A. The scalar transport equation}

Consider the (already diagonal) 1-D scalar transport equation

$$
\frac{\partial x}{\partial t}=\frac{\partial x}{\partial \zeta}
$$

with $x \in \mathbb{R}, \zeta \in(0,1)$ and boundary condition

$$
u(t)=x(t, 1) .
$$

This is a trivial example and as pointed out in Remark 3.2 the conditions of Proposition 3.1 are automatically satisfied. In this example $P_{1}=1$ and $W_{B}=W_{B, 1}=\left[\begin{array}{ll}1 & 0\end{array}\right]$. Now define as target system

$$
\frac{\partial z}{\partial t}=\frac{\partial z}{\partial \zeta}-c z
$$

with $z \in \mathbb{R}, c>0$ and homogeneous boundary condition

$$
0=z(t, 1),
$$

In this case, since $W_{B}=W_{B, 1}$ the conditions of Proposition 3.1 are automatically satisfied and the boundary controller can be directly computed using (7)

$$
u(t)=-\left[\begin{array}{ll}
1 & 0
\end{array}\right]\left[\begin{array}{c}
\left(\alpha e^{\frac{c}{\lambda}}-1\right) x(t, 1) \\
(\alpha-1) x(t, 0)
\end{array}\right]=\left(1-\alpha e^{\frac{c}{\lambda}}\right) x(t, 1),
$$

and from (26 we recover the coordinate transformation $z(t, 1)=M(1) x(t, 1)$,

$$
x(t, 1)=\alpha e^{\frac{c}{\lambda}} x(t, 1)=z(t, 1)=0 .
$$

It is interesting to notice that the target system and the openloop system are equivalent when considering the proposed coordinate transformation. The zero boundary condition is a well known trivial result [14], and just shows that by injecting zero at the input boundary the energy of the system converges and remains at zero.

\section{B. The vibrating string (wave equation)}

Consider now the non-trivial clamped vibrating string

$$
\frac{\partial \tilde{x}}{\partial t}=\left[\begin{array}{ll}
0 & 1 \\
1 & 0
\end{array}\right] \frac{\partial \tilde{x}}{\partial \zeta}
$$

with $\tilde{x} \in \mathbb{R}^{2}$, where $\tilde{x}_{1}$ and $\tilde{x}_{2}$ correspond respectively to the velocity and the strain of the string, $\zeta \in(0,1)$ and boundary conditions

$$
\begin{aligned}
u(t) & =\tilde{x}_{2}(t, 1), \\
0 & =\tilde{x}_{1}(t, 0) .
\end{aligned}
$$

Hence, the string is clamped at $\zeta=0$ and actuated at $\zeta=1$. In this case $P_{1}=\left[\begin{array}{ll}0 & 1 \\ 1 & 0\end{array}\right], W_{B, 1}=\left[\begin{array}{llll}0 & 1 & 0 & 0\end{array}\right]$ and $W_{B, 2}=$ $\left[\begin{array}{llll}0 & 0 & 1 & 0\end{array}\right]$. Define as target system

$$
\frac{\partial \tilde{z}}{\partial t}=\left[\begin{array}{ll}
0 & 1 \\
1 & 0
\end{array}\right] \frac{\partial \tilde{z}}{\partial \zeta}-c I \tilde{z}
$$

with $\tilde{z} \in \mathbb{R}^{2}, c>0$ and homogeneous boundary conditions

$$
\begin{aligned}
& 0=\tilde{z}(t, 1), \\
& 0=\tilde{z}(t, 0) .
\end{aligned}
$$


A diagonalizing transformation is given by

$$
\begin{aligned}
& x_{1}=\tilde{x}_{1}+\tilde{x}_{2} \\
& x_{2}=\tilde{x}_{1}-\tilde{x}_{2}
\end{aligned}
$$
obtained with $T=\left[\begin{array}{cc}\frac{1}{2} & \frac{1}{2} \\ \frac{1}{2} & -\frac{1}{2}\end{array}\right]$. Hence a diagonal representa-
tion of (29) is

$$
\frac{\partial x}{\partial t}=\left[\begin{array}{cc}
1 & 0 \\
0 & -1
\end{array}\right] \frac{\partial x}{\partial \zeta}
$$

with $x \in \mathbb{R}^{2}$ and boundary conditions

$$
\begin{aligned}
2 u(t) & =x_{1}(t, 1)-x_{2}(t, 1), \\
0 & =x_{1}(t, 0)+x_{2}(t, 0) .
\end{aligned}
$$

The diagonal target system is given by

$$
\frac{\partial z}{\partial t}=\left[\begin{array}{cc}
1 & 0 \\
0 & -1
\end{array}\right] \frac{\partial z}{\partial \zeta}-c I z
$$

with $z \in \mathbb{R}^{2}$ and homogeneous boundary conditions

$$
\begin{aligned}
& 0=z_{1}(t, 1)-z_{2}(t, 1), \\
& 0=z_{1}(t, 0)+z_{2}(t, 0) .
\end{aligned}
$$

In order to compute the control using Proposition 3.1 we need to select the constants $\alpha_{1}$ and $\alpha_{2}$ such that the coordinate transformation is invertible. From (20) and (22) we can rewrite (35) as

$$
z_{1}(t, 0)+z_{2}(t, 0)=0 \Leftrightarrow \alpha_{1} x_{1}(t, 0)+\alpha_{2} x_{2}(t, 0)=0 .
$$

This has to be satisfied for all $x_{1}, x_{2}$ satisfying (33). Thus we see that is true if $\alpha_{1}=\alpha_{2}=\alpha$. The control can be computed from (7) or using (34)

$$
\alpha e^{\frac{c}{\lambda_{1}}}\left(\tilde{x}_{1}(1)+\tilde{x}_{2}(1)\right)-\alpha e^{\frac{c}{\lambda_{2}}}\left(\tilde{x}_{1}(1)-\tilde{x}_{2}(1)\right)=0
$$

from where we obtain

$$
u(t)=\tilde{x}_{2}(t, 1)=\left(\frac{e^{\frac{c}{\lambda_{2}}}-e^{\frac{c}{\lambda_{1}}}}{e^{\frac{c}{\lambda_{2}}}+e^{\frac{c}{\lambda_{1}}}}\right) \tilde{x}_{1}(t, 1)
$$

and recalling that $\lambda_{1}=1$ and $\lambda_{2}=-1$ the stabilizing boundary controller is given by

$$
u(t)=-\left(\frac{e^{c}-e^{-c}}{e^{c}+e^{-c}}\right) \tilde{x}_{1}(t, 1)=-\tanh (c) \tilde{x}_{1}(t, 1) .
$$

It is well known that a passive damper of this kind exponentially stabilizes the system. It is interesting to notice that the derived controller can either be interpreted as the connection of a damper to the extreme $\zeta=1$ of the string, or as the mapping of the conservative PHS formulation of the string equation into the dissipative PHS (30). Another interesting aspect is that the controller is comparable to the one deduced in [6], where the state variable is the position and an integral Volterra coordinate transformation is used.

\section{Conclusion}

A backstepping approach has been used to develop a boundary controller for linear PHS with constant parameters. A dissipative PHS, i.e., a PHS with an in-domain dissipation term, with homogenoeus boundary conditions has been used as target system. A coordinate transformation based on a multiplicative operator has been deduced by diagonalizing the open-loop and the target systems. The associated matching condition is an algebraic equation, which is simpler than the usual PDEs associated to the kernel conditions in standard backstepping. Since the controller has been developed for a general linear PHS it is applicable to a large class of physical systems, as for instance transport, beam and wave equations. The result have been illustrated on the examples of a transport equation and a vibrating string on a 1D spatial domain.

Future work will deal with linear PHS with non-constant parameters and the design of adaptive controllers.

\section{REFERENCES}

[1] H. Fattorini, "Boundary control systems," SIAM Journal on Control, vol. 6 , no. 3, pp. 349-385, 1968.

[2] A. Smyshlyaev and M. Krstic, "Closed-form boundary state feedbacks for a class of 1-d partial integro-differential equations," IEEE Transactions on Automatic Control, vol. 49, no. 12, pp. 2185-2202, Dec 2004.

[3] - "Backstepping observers for a class of parabolic PDEs," Systems \& Control Letters, vol. 54, no. 7, pp. 613 - 625, 2005.

[4] M. Krstic and A. Smyshlyaev, Boundary Control of PDEs: A Course on Backstepping Designs, ser. Advances in Design and Control.

[5] T. Meurer and A. Kugi, "Tracking control for boundary controlled parabolic PDEs with varying parameters: Combining backstepping and differential flatness," Automatica, vol. 45, no. 5, pp. 1182 - 1194, 2009.

[6] A. Smyshlyaev, E. Cerpa, and M. Krstic, "Boundary stabilization of a 1-d wave equation with in-domain antidamping," SIAM Journal on Control and Optimization, vol. 48, no. 6, pp. 4014-4031, 2010.

[7] J.-M. Wang, L.-L. Su, and H.-X. Li, "Stabilization of an unstable reaction-diffusion PDE cascaded with a heat equation," Systems \& Control Letters, vol. 76, pp. 8 - 18, 2015.

[8] J. Auriol and F. D. Meglio, "Minimum time control of heterodirectional linear coupled hyperbolic PDEs," Automatica, vol. 71, pp. 300 - 307, 2016.

[9] A. Diagne, M. Diagne, S. Tang, and M. Krstic, "Backstepping stabilization of the linearized Saint-Venant-Exner model," Automatica, vol. 76, pp. $345-354,2017$.

[10] A. van der Schaft and B. Maschke, "Hamiltonian formulation of distributed-parameter systems with boundary energy flow," Journal of Geometry and Physics, vol. 42, no. 1-2, pp. 166 - 194, 2002.

[11] Y. Le Gorrec, H. Zwart, and B. Maschke, "A semigroup approach to port hamiltonian systems associated with linear skew symmetric operator," 16th International Symposium on Mathematical Theory of Networks and Systems (MTNS 2004), 2004.

[12] — - "Dirac structures and boundary control systems associated with skew-symmetric differential operators," SIAM Journal on Control and Optimization, vol. 44, no. 5, pp. 1864-1892, 2005.

[13] J. A. Villegas, "A port-Hamiltonian approach to distributed parameter systems," Ph.D. dissertation, Universiteit Twente, 2007.

[14] B. Jacob and H. Zwart, Linear Port-Hamiltonian Systems on Infinitedimensional Spaces, ser. Operator Theory: Advances and Applications. Basel, Switzerland: Birkhäuser, 2012, vol. 223.

[15] H. Zwart, Y. L. Gorrec, B. Maschke, and J. Villegas, "Well-posedness and regularity of hyperbolic boundary control systems on a onedimensional spatial domain," ESAIM: Control, Optimisation and Calculus of Variations, vol. 16, no. 4, pp. 1077-1093, 2010.

[16] J. Villegas, H. Zwart, Y. Le Gorrec, B. Maschke, and A. van der Schaft, "Stability and stabilization of a class of boundary control systems," in Decision and Control, 2005 and 2005 European Control Conference. CDC-ECC '05. 44th IEEE Conference on, dec. 2005, pp. 3850-3855. 
[17] J. Villegas, H. Zwart, Y. Le Gorrec, and B. Maschke, "Exponential stability of a class of boundary control systems," IEEE Transactions on Automatic Control, vol. 54, pp. 142-147, 2009.

[18] H. Ramirez, Y. L. Gorrec, A. Macchelli, and H. Zwart, "Exponential stabilization of boundary controlled port-Hamiltonian systems with dynamic feedback," IEEE Transactions on Automatic Control, vol. 59, no. 10, pp. 2849-2855, Oct 2014

[19] B. Augner and B. Jacob, "Stability and stabilization of infinitedimensional stability and stabilization of infinite-dimensional linear port-hamiltonian systems," Evolution Equations and Control Theory, DOI: 10.3934/eect.2014.3.207, vol. 3, no. 2, pp. 207-229, December 2014.

[20] A. Macchelli, Y. L. Gorrec, H. Ramirez, and H. Zwart, "On the synthesis of boundary control laws for distributed port Hamiltonian systems," IEEE Transactions on Automatic Control, vol. PP, no. 99, pp. $1-1,2016$

[21] B. Jacob, K. Morris, and H. Zwart, " $C_{0}$-semigroups for hyperbolic partial differential equations on a one-dimensional spatial domain," $J$. Evol. Equ., vol. 15, no. 2, pp. 493-502, 2015.

[22] A. J. van der Schaft, L2-Gain and Passivity Techniques in Nonlinear Control, 2nd ed. New York, USA: Springer-Verlag, 2000. 